\title{
Hamiltonian Structures and Lax Equations Generated by Matrix Differential Operators with Polynomial Dependence on a Parameter
}

\author{
L. A. Dickey \\ Leningradsky av. 28, fl. 59, SU-125040 Moscow, USSR
}

\begin{abstract}
We investigate a general set of equations which can be studied by the inverse scattering method.
\end{abstract}

In the present paper we study Hamiltonian structures and equations of the Lax type which arise in connection with a first-order linear differential operator $L=\partial$ $+U_{0}+U_{1} \zeta+\ldots+U_{n} \zeta^{n}+A \zeta^{n+1}$, where $\partial=\frac{d}{d x} ; U_{i}$ are matrices, and $A$ is a constant diagonal matrix. The case $n=0$ is well-known.

Much attention is also paid to the Lagrangian formalism for these equations and its connection with the Hamiltonian approach. Two different Lagrangians are found.

\section{Lie Algebra $R_{-}$}

$\mathscr{A}$ is a differential algebra consisting of the polynomials (with real coefficients) in matrix elements of some matrices $U_{0}, U_{1}, \ldots, U_{n}$ and their derivatives with respect to $x$ of arbitrary orders. Thus elements of $U_{i}$ are independent generators of this differential algebra: $R_{-}$is a Lie algebra consisting of the matrix bundles, $X=\sum_{i=0}^{n} X_{i} \zeta^{-i-1}$, where elements of $X_{i}$ belong to $\mathscr{A}$, and $\zeta$ is a formal parameter. In this algebra the commutator is introduced by

$$
[X, Y]_{3}=\overline{[X, Y]\left(\zeta^{n+1}+\jmath\right)} \text {. }
$$

Here $[$,$] is the ordinary commutator of matrices, z$ is a fixed real number, the bar symbolizes cutting out a segment of a series in $\zeta^{-1}$ from $\zeta^{-1}$ to $\zeta^{-n-1}$.

It is clear that the commutator (1.1) is a linear combination of two commutators : from $[X, Y]$ we cut out either the second half of the powers of $\zeta^{-1}$ (from $\zeta^{-n-2}$ to $\zeta^{-2 n-2}$ ) and multiply it by $\zeta^{n+1}$, or the first half (from $\zeta^{-1}$ to $\zeta^{-n-1}$ ). So we have a family of commutators depending on the parameter $z$. 
Lemma. The expression (1.1) has all the properties of a commutator.

The simplest proof of this lemma can be obtained if we consider $R_{\text {_ }}$ as a module over the ring $\mathbb{R}\left(\zeta^{-1}\right)$ of series in $\zeta^{-1}$ with real coefficients, where $\zeta^{-n-2}$ is identified with $z^{-1}$. Then (1.1) is simply $3[X, Y]$.

\section{Dual Space $\boldsymbol{R}_{+}$}

$R_{+}$consists of the matrix bundles $M=\sum_{i=0}^{n} M_{i} \zeta^{i}$, where the elements of $M_{i}$ belong to $\mathscr{A}$. One of the elements of $R_{+}$is $U=\sum_{0}^{n} U_{i} \zeta^{i}$, where $U_{i}$ are the matrices whose elements were taken as the generators of $\mathscr{A}$. The space $R_{+}$is dual to $R_{-}$relative to the coupling

$$
(M, X)=\int \operatorname{tr} \sum_{0}^{n} M_{i} X_{i} d x=\int \operatorname{tr} \operatorname{res}(M X) d x,
$$

where res denotes the coefficient in $\zeta^{-1}: \operatorname{res} \sum A_{i} \zeta^{i}=A_{-1}$. [Here the integral is formal; it means the projection of $\mathscr{A}$ on the quotient-space $\mathscr{A}=\mathscr{A} / \partial \mathscr{A}$. In nonformal theory it is possible to use here any concept of the integral provided that the property $\int f^{\prime} d x=0$ holds for every $f(x)$ belonging to the function class under consideration.]

Let us define $[M, X]_{3}^{*}$ for $M \in R_{+}, X \in R_{-}$:

$$
\left(M,[X, Y]_{3}\right)=\left([M, X]_{3}^{*}, Y\right) \text {. }
$$

It is easy to check that

$$
[M, X]_{3}^{*}=\tilde{[M, X]\left(\zeta^{n+1}+z\right)}
$$

The wavy line symbolizes cutting out a segment of a series in $\zeta$ from $\zeta^{0}$ to $\zeta^{n}$.

To every $M \in R_{+}$we assign the differentiation in $\mathscr{A}$ (a "vector field"):

$$
\xi_{M}=\sum_{i j k \ell} M_{i, j k}^{(\ell)} \partial / \partial U_{i, j k}^{(\ell)}=\operatorname{tr} \sum_{i \ell} M_{i}^{(\ell)} \partial / \partial U_{i}^{(\ell) *}
$$

(the asterisk denotes the transpose of a matrix). These differentiations commute with $\partial$, and therefore can be considered in $\tilde{\mathscr{A}}$. We shall also apply them to the elements of $R_{ \pm}$(to every element of every matrix). It is obvious that $\xi_{M} U=M$. Now we denote

$$
L=\partial+U+A \zeta^{n+1},
$$

where $A$ is a constant diagonal matrix with different diagonal elements. Let us consider a mapping $R_{-} \rightarrow R_{+}$:

$$
\begin{aligned}
& X \in R_{-} \mapsto M_{X}=\tilde{[L, X]\left(\zeta^{n+1}+z\right) \in R_{+}} \text {, } \\
& M_{X}=M_{X}^{(1)}+z M_{X}^{(2)} \text {. }
\end{aligned}
$$

We shall denote the vector field $\xi_{M_{X}}$ more simply by $\xi_{X}$. The lemma below can easily be proved by a direct calculation:

Lemma. For two vector fields $\xi_{X}$ and $\xi_{Y}$, we have

$$
\left[\xi_{X}, \xi_{Y}\right]=\xi_{[X, Y]_{3}+\xi_{X} Y-\xi_{Y} X} \text {. }
$$




\section{Symplectic Form}

On vector fields of the type $\xi_{X}$ a form can be defined:

$$
\begin{aligned}
\omega\left(\xi_{X}, \xi_{Y}\right) & =\operatorname{tr} \operatorname{res} \int M_{X} Y d x=\operatorname{tr} \operatorname{res} \int\left(\zeta^{n+1}+z\right)[L, X] Y d x \\
& =\omega_{1}\left(\xi_{X}, \xi_{Y}\right)+z \omega_{2}\left(\xi_{X}, \xi_{Y}\right) .
\end{aligned}
$$

Proposition. The form $\omega$ is closed.

Proof. For any three vector fields we have (the summation extends over the cyclic permutations of $X, Y$, and $Z$ ):

$$
\begin{aligned}
d \omega\left(\xi_{X}, \xi_{Y}, \xi_{Z}\right)= & \sum\left\{\xi_{X} \omega\left(\xi_{Y}, \xi_{Z}\right)-\omega\left(\left[\xi_{X}, \xi_{Y}\right], \xi_{Z}\right)\right\} \\
= & \sum \operatorname{tr} \operatorname{res} \int\left\{\left(\zeta^{n+1}+\jmath\right)\left(\left[M_{X}, Y\right] Z+\left[L, \xi_{X} Y\right] Z+[L, Y] \xi_{X} Z\right)\right. \\
& \left.+M_{Z}[X, Y]\left(\zeta^{n+1}+\jmath\right)+M_{Z}\left(\xi_{X} Y-\xi_{Y} X\right)\right\} d x \\
= & 2 \sum \operatorname{tr} \operatorname{res} \int\left(\zeta^{n+1}+\jmath\right)\left[M_{Z}, X\right] Y d x \\
= & 2 \sum \operatorname{tr} \operatorname{res} \int\left(\zeta^{n+1}+\jmath\right)\left[\left(\zeta^{n+1}+\jmath\right)[L, Z], X\right] Y d x \\
= & 2 \sum \operatorname{tr} z \operatorname{res} \int\left\{\zeta^{n+1}\left[\zeta^{n+1}[L, Z], X\right] Y+\jmath\left[\zeta^{n+1}[L, Z], X\right] Y\right. \\
& \left.+\jmath\left[\zeta^{n+1}[\tilde{L}, Z], X\right] Y+\jmath^{2}[[L, Z], X] Y\right\} d x \\
= & 2 \operatorname{tr} \operatorname{res} \int\left(\zeta^{2 n+2}+\jmath \zeta^{n+1}+z^{2}\right)[[L, Z], X] Y d x .
\end{aligned}
$$

Now

$$
\begin{aligned}
\int \sum \operatorname{tr}[[L, Z], X] Y d x & =-\int \sum \operatorname{tr}[Y, X][L, Z] d x \\
& =-\int \sum \operatorname{tr}[Y, X]\left([L-\partial, Z]+Z^{\prime}\right) d x \\
& =\int \sum \operatorname{tr}(L-\partial)[[Y, X], Z] d x-\int \operatorname{tr}([Y, X] Z)^{\prime} d x=0
\end{aligned}
$$

as stated.

Remark. The construction of $\omega$ slightly differs from that of Kirillov-Kostant, since the mapping $M_{X}: R_{-} \rightarrow R_{+}$(2.3) does not coincide with the coadjoint repre-

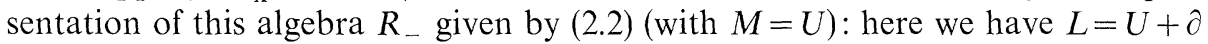
$+A \zeta^{n+1}$ instead of $U$.

\section{Hamiltonian Vector Fields and Equations}

To every functional $\tilde{f}=\int f d x \in \tilde{\mathscr{A}}$ we assign a vector field $\xi_{f}$ such that for any vector field $\xi_{Y}$ the relation $\xi_{Y} \tilde{f}=-\omega\left(\xi_{f}, \xi_{Y}\right)$ holds. We have

$$
\begin{aligned}
\xi_{Y} \tilde{f} & =\int \operatorname{tr} \sum_{i \ell} M_{Y i}^{(\ell)} \frac{\partial f}{\partial U_{i}^{(\ell) *}} d x=\int \operatorname{tr} \sum_{i} M_{Y i} \frac{\delta f}{\delta U_{i}^{*}} d x \\
& =\operatorname{tr} \operatorname{res} \int M_{Y} X_{f} d x=-\omega\left(\xi_{X_{f}}, \xi_{Y}\right),
\end{aligned}
$$

where $X_{f}=\sum_{0}^{n} \delta f / \delta U_{i}^{*} \cdot \zeta^{-i-1}$. Thus we have

Lemma. The Hamiltonian vector field corresponding to a functional $\tilde{f}=\int f d x$ is

$$
\xi_{f}=\xi_{M_{X_{f}}} .
$$


It should be noted that (4.1) can also be written as

$$
\delta \tilde{f}=\int \operatorname{tr} \operatorname{res} X_{f} \delta U d x=-i\left(\xi_{X_{f}}\right) \omega .
$$

It may also be useful to write

$$
\delta f=\operatorname{tr} \operatorname{res} X_{f} \delta U+\partial \omega_{s},
$$

where $\omega_{s}$ is a 1 -form of $\delta U, \delta U^{\prime}, \ldots$. This form is important for the theory of stationary equations (see [1]).

Now we define the Poisson bracket of two functionals as

$$
\{\tilde{f}, \tilde{g}\}=\xi_{f} \tilde{g}=\{\tilde{f}, \tilde{g}\}_{1}+z\{\tilde{f}, \tilde{g}\}_{2} .
$$

The definition involves the parameter $z$, i.e. here a bundle of Poisson brackets is defined.

Let us write the differential equation corresponding to a Hamiltonian vector field :

$$
\dot{U}=M_{X_{f}},
$$

or, in more detail,

$$
\begin{aligned}
\dot{U}_{i}= & \sum_{m=n-i}^{n}\left[U_{i+m-n}, \frac{\delta f}{\delta U_{m}^{*}}\right]+\left(\frac{\delta f}{\delta U_{n-i}^{*}}\right)^{\prime} \\
& +z \sum_{m=0}^{n-i}\left[U_{i+m+1}, \frac{\delta f}{\delta U_{m}^{*}}\right] ; \quad i=0, \ldots, n ; \quad U_{n+1}=A .
\end{aligned}
$$

There are two extreme cases here:

$$
\dot{U}_{i}=\sum_{m=n-i}^{n}\left[U_{i+m-n}, \frac{\delta f}{\delta U_{m}^{*}}\right]+\left(\frac{\delta f}{\delta U_{n-i}^{*}}\right)^{\prime}, \quad i=0, \ldots, n,
$$

and

$$
\dot{U}_{i}=\sum_{m=0}^{n-i}\left[U_{i+m+1}, \frac{\delta f}{\delta U_{m}^{*}}\right], \quad i=0, \ldots, n ; \quad U_{n+1}=A
$$

\section{Resolvent}

Now we shall construct Lax equations. They correspond to special Hamiltonians connected with a resolvent of the differential operator $L$.

The resolvent is a formal series

$$
\mathscr{R}=\sum_{0}^{\infty} R_{k} \zeta^{-k-1}
$$

where $R_{k}$ are matrices with elements of $\mathscr{A}$ which satisfies the equation

$$
[L, \mathscr{R}]=0 \text {. }
$$

Let

$$
\mathscr{R}_{p}=\sum_{k=0}^{n} R_{p+k} \zeta^{-k-1} \in R_{-}
$$


and

$$
\tilde{\mathscr{R}}=\sum_{\ell=0}^{\infty} z^{-\ell} \mathscr{R}_{-r_{0}+(n+1) \ell},
$$

where $r_{0}$ is a fixed integer between 0 and $n . R_{k}$ with a negative subscript is zero. It is clear that

$$
\mathscr{R}=\left.\tilde{\mathscr{R}}\right|_{3}=\zeta^{n+1} \cdot \zeta^{r_{0}} \text {, }
$$

and $\tilde{\mathscr{R}}$ belongs to the ring $R_{-}\left(\left(z^{-1}\right)\right)$ of formal series in $z^{-1}$ with coefficients belonging to $R_{-}$. We call $\tilde{\mathscr{R}}$ a polarization of the resolvent $\mathscr{R}$. Depending on $r_{0}$ there exist $n+1$ different polarizations of a given resolvent.

The mapping $M$ defined earlier in $R_{-}$can be defined in $R_{-}\left(\left(3^{-1}\right)\right)$ as well:

$$
M_{\tilde{\mathscr{R}}}=\tilde{[L, \mathscr{R}]}\left(\zeta^{n+1}+\not\right) \in R_{+}\left(\left(z^{-1}\right)\right) \text {. }
$$

Proposition. The polarization of the resolvents form the kernel of the mapping $M_{\mathscr{R}}$, i.e. (5.2) is equivalent to

$$
\left.M_{\tilde{\mathscr{R}}}=\operatorname{mL}_{[L, \mathscr{R}]} \boldsymbol{\zeta}^{n+1}+\mathfrak{\zeta}\right)=0 .
$$

Proof. We have

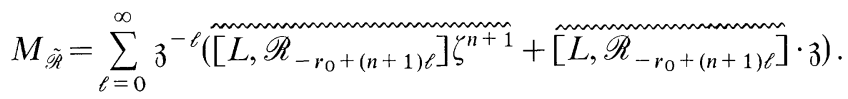

Now we substitute $z=\zeta^{n+1}$ :

$$
\begin{aligned}
M_{\left.\tilde{\mathscr{R}}\right|_{3}=\zeta^{n+1}} & =\sum_{\ell=0}^{\infty} \zeta^{-\ell(n+1)}\left(\left[\tilde{L}, \mathscr{R}_{-r_{0}+(n+1) \ell}\right] \zeta^{n+1}+\left[L, \mathscr{R}_{-r_{0}+(n+1) \ell}\right] \cdot \zeta^{n+1}\right) \\
& =\sum_{\ell=0}^{\infty} \zeta^{-\ell(n+1)}\left[L, \mathscr{R}_{-r_{0}+(n+1) \ell}\right] \zeta^{n+1}=[L, \mathscr{R}] \zeta^{-r_{0}+n+1} .
\end{aligned}
$$

Thus $[L, \mathscr{R}]=0$ is equivalent to $\left.M_{\mathscr{R}}\right|_{z=\zeta^{n+1}}=0$. The substitution $z=\zeta^{n+1}$ transforms terms corresponding to different powers of $z^{-1}$ into different segments of the series in $\zeta^{-1}$, and therefore $\left.M_{\tilde{\mathscr{R}}}\right|_{3=\zeta^{n+1}}=0$ is equivalent to $M_{\tilde{\mathscr{R}}}=0$.

$M_{\tilde{\mathscr{R}}}$ depends on $z$ linearly: $M_{\tilde{\mathscr{R}}}=M_{\mathscr{\mathscr { R }}}^{(1)}+z M_{\mathscr{\mathscr { R }}}^{(2)}$.

Lemma. Equation (5.2) is equivalent to recurrence relations

$$
M_{\mathscr{R}_{-r_{0}+(n+1) \ell}^{(1)}}^{(2)}+M_{\mathscr{R}_{-r_{0}+(n+1)(\ell+1)}^{(2)}}^{(2)}=0 .
$$

Proof. It is sufficient to substitute $\tilde{\mathscr{R}}=\sum_{0}^{\infty} z^{-\ell} \mathscr{R}_{-r_{0}+(n+1) \ell}$ into $M_{\mathscr{R}}^{(1)}+z^{M_{\mathscr{R}}^{(2)}}=0$ and equate the terms with the same powers of $\mathfrak{z}$.

Corollary. For any natural $r$ we have

$$
M_{\mathscr{R}_{r}}^{(1)}+M_{\mathscr{R}_{r+n+1}}^{(2)}=0 .
$$

\section{Existence of Resolvents}

Let us consider the equation

$$
L \varphi=\lambda \varphi
$$


The column vector $\varphi$ is sought as a formal series $\sum_{0}^{\infty} \varphi_{r} \zeta^{-r}$. The scalar $\lambda$ is also an unknown series : $\lambda=\sum_{-1-n}^{\infty} \lambda_{r} \zeta^{-r}$. The elements of the vectors $\varphi_{r}$ and the coefficients
$\lambda_{r}$ belong to $\mathscr{A}$.

Lemma 1. If $m$ is the dimension of the vector space, then there are $m$ linearly independent solutions $\varphi$, the scalar $\lambda$ being completely determined.

Proof. Equation (6.1) is equivalent to recurrence relations of the form

$$
\left(A-\lambda_{-n-1}\right) \varphi_{r}+\lambda_{r-n-1} \varphi_{0}+F_{r}\left(\varphi_{0}, \ldots, \varphi_{r-1}, \lambda_{-n-1}, \ldots, \lambda_{r-n-2}\right)=0,
$$

where $F_{r}$ is a vector-function. The initial recurrence formula is $\left(A-\lambda_{-n-1}\right) \varphi_{0}=0$. Hence $\lambda_{-n-1}$ is one of the eigenvalues of the diagonal matrix $A$, and $\varphi_{0}$ is the corresponding eigenvector. For the solvability of (6.2) it is necessary and sufficient that

$$
\lambda_{r-n-1}\left(\varphi_{0}, \varphi_{0}\right)+\left(F_{r}, \varphi_{0}\right)=0 .
$$

This determines $\lambda_{r-n-1}$, and then (6.2) gives $\varphi_{r}$ to within a multiple of $\varphi_{0}$; we can specify this solution by the requirement that $\varphi_{r}, r>0$ contain no constants in any of its coordinates.

From $m$ solutions a matrix $\Phi=\left(\varphi_{k s}\right)$ can be formed (here $s$ is the number of a column, i.e. of a solution, and $k$ is the number of a coordinate of this vector). Here $\Phi=\sum_{0}^{\infty} \Phi_{r} \zeta^{-r}$, where $\Phi_{0}$ is a constant invertible matrix; hence $\Phi$ is invertible as a formal series in $\zeta^{-1}$. Let $\Psi=\Phi^{-1} . \Phi$ satisfies the equation

$$
\Phi^{-1}+U \Phi+A \zeta^{n+1} \Phi=\Phi \Lambda, \quad \Lambda=\left(\begin{array}{lll}
\lambda^{(1)} & & \\
& \ddots & \\
& & \lambda^{(m)}
\end{array}\right)
$$

(the superscript in $\lambda^{(i)}$ is the number of the solution). Here $\Psi$ satisfies

$$
-\Psi^{\prime}+\Psi U+\Psi A \zeta^{n+1}=\Lambda \Psi,
$$

i.e. the conjugate equation. Let $\Psi=\left(\psi_{s k}\right)$ (the number of a row $s$ is a number of a solution of the conjugate equation).

Proposition. The matrices

$$
\mathscr{R}^{(s)}=\left(\mathscr{R}_{k \ell}^{(s)}\right)=\left(\varphi_{k s} \psi_{s \ell}\right), \quad s=1, \ldots, m
$$

are resolvents. The general form of a resolvent is $\sum_{s=1}^{m} \dot{\mathscr{A}}^{(s)} \mathscr{R}^{(s)}$, where $\mathscr{A}^{(s)}$ are series in $\zeta^{-1}$ with constant coefficients.

Proof. From the equations

$$
\begin{gathered}
\varphi^{\prime}+\left(U+A \zeta^{n+1}\right) \varphi=\lambda \varphi, \\
-\psi^{\prime}+\psi\left(U+A \zeta^{n+1}\right)=\lambda \psi,
\end{gathered}
$$

for column vectors $\varphi^{(s)}=\left(\varphi_{k s}\right)$ and row vectors $\psi^{(s)}=\left(\psi_{s k}\right)$, we obtain for $\mathscr{R}=\varphi \cdot \psi$ the required equation without any difficulty. To prove the second assertion we use the following lemma. 
Lemma 2. If the diagonal elements of any of the matrices $R_{k}$ of a resolvent $\mathscr{R}$ do not contain constants, then this resolvent is trivial zero.

Proof. We can suppose that $R_{0} \neq 0$. The first $n+2$ equations defining a resolvent are

$$
\begin{gathered}
{\left[A, R_{0}\right]=0,} \\
{\left[A, R_{1}\right]+\left[U_{n}, R_{0}\right]=0 .} \\
\ldots \ldots \ldots \ldots \ldots \ldots \ldots \ldots . \\
{\left[A, R_{n}\right]+\ldots+\left[U_{1}, R_{0}\right]=0,} \\
R_{0}^{\prime}+\left[A, R_{n+1}\right]+\ldots+\left[U_{0}, R_{0}\right]=0 .
\end{gathered}
$$

The first of these equations implies that $R_{0}$ is diagonal. We must show that $R_{0}^{\prime}=0$. Let $V=(L-\partial) \zeta^{-n-1}=U \zeta^{-n-1}+A, S=\mathscr{R} \cdot \zeta$. From (6.7) it is seen that the senior terms in $\zeta$ of the commutator $[V, S]$ (from $\zeta^{0}$ to $\zeta^{-n}$ ) vanish while the next term has vanishing nondiagonal elements. Let us show that the diagonal elements of this term also vanish, which will imply that $R_{0}^{\prime}=0$. We have

$$
\begin{aligned}
0 & =\frac{1}{p+1} \operatorname{tr}\left[V^{p+1}, S\right]=\operatorname{tr} V^{p}[V, S] \\
& =\operatorname{tr} A^{p}[V, S]+\operatorname{tr} O\left(\zeta^{-1}\right) \cdot[V, S] .
\end{aligned}
$$

Let us consider the coefficient in $\zeta^{-n-1}$. The second term does not involve $\zeta^{-n-1}$, and for any $p$ we obtain $\left.\operatorname{tr} A^{p}[V, S]\right|_{\zeta-n-1}=0$. Hence the diagonal elements of $[V, S]_{\zeta^{-n-1}}$ vanish. Thus $R_{0}^{\prime}=0$ and $R_{0}=0$, since $R_{0}$ does not contain constants.

To complete the proof of the proposition we note that the resolvents (6.5) have only one constant, namely the unity in the $s^{\text {th }}$ place of the diagonal. It is possible to obtain an arbitrary combination of constants on the diagonal of a resolvent and thus an arbitrary resolvent by choosing appropriately series $\mathscr{A}^{(s)}$.

The resolvents form a ring (if $\mathscr{R}_{a}$ and $\mathscr{R}_{b}$ commute with $L$ so do their sum and product). We have $\operatorname{tr} \mathscr{R}=$ const, which is easily seen from the equation for a resolvent. In particular, for $\mathscr{R}^{(s)}$ from (6.5) we have tr $\mathscr{R}^{(s)}=1$. Further, $\mathscr{R}^{(s) 2}=\mathscr{R}^{(s)}$ since $\mathscr{R}^{(s) 2}$ and $\mathscr{R}^{(s)}$ have the same constants. Hence $\mathscr{R}^{(s)}$ are projectors. Thus $\mathscr{R}=\sum a^{(s)} \mathscr{R}^{(s)}$ gives the spectral decomposition of the resolvent.

\section{Variational Theorem}

Theorem. For the resolvent $\mathscr{R}=\varphi \cdot \psi$ of $(6.5)$, the equation

$$
\delta \operatorname{tr} L_{\zeta} \mathscr{R}=\operatorname{tr}(\delta U \cdot \mathscr{R})_{\zeta}+\partial \operatorname{tr}\left(-\delta \varphi \cdot \psi_{\zeta}+\varphi_{\zeta} \cdot \delta \psi\right)
$$

holds. (The subscript $\zeta$ denotes the derivative.)

The proof of this theorem can easily be obtained from (6.6). We omit it because it is the same as in the case $n=0$ (see [1]).

\section{Corollary.}

$$
\left.\frac{\delta}{\delta U_{k}^{*}} \int \operatorname{tr} L_{\zeta} \mathscr{R} d x\right|_{r}=(-r+1) R_{r+k-1}
$$

(the subscript $r$ denotes the coefficient in $\zeta^{-r}$ ). 
It should be noted that on the left-hand side of this equation there are $R_{\ell}$ ending with $R_{r+n}$, and the right-hand side contains $R_{\ell}$ with smaller numbers only.

\section{Involutive System of Hamiltonians}

Let

$$
H_{r}=\left.\int \operatorname{tr}\left(U+\zeta^{n+1} A\right)_{\zeta} \mathscr{R} d x\right|_{r},
$$

where $\mathscr{R}$ is a linear combination of resolvents (6.5) with constant coefficients.

Theorem. Functionals $H_{r} \in \mathscr{A}$ are in involution with respect to the Poisson brackets $\{,\}_{1}$ and $\{,\}_{2}$ defined in Sect. 4 .

Proof. We have

$$
X_{H_{r}}=\sum_{0}^{n} \frac{\delta H_{r}}{\delta U_{k}^{*}} \zeta^{-k-1}=(-r+1) \sum_{0}^{n} R_{r+k-1} \zeta^{-k-1}=(-r+1) \mathscr{R}_{r-1} .
$$

It is necessary to show that

$$
\int \operatorname{tr} \operatorname{res} M_{\mathscr{R}_{r}}^{(1)} \cdot \mathscr{R}_{s} d x=\int \operatorname{tr} \operatorname{res} M_{\mathscr{R}_{r}}^{(2)} \cdot \mathscr{R}_{s} d x=0
$$

holds for any $r, s$. We can use the recurrence relation (5.7) and the skew symmetry of the Poisson brackets :

$$
\begin{aligned}
\int \operatorname{tr} \operatorname{res} M_{\mathscr{R}_{r}}^{(1)} \cdot \mathscr{R}_{s} d x & =-\int \operatorname{tr} \operatorname{res} M_{\mathscr{R}_{r+n+1}}^{(2)} \cdot \mathscr{R}_{s} d x \\
& =\int \operatorname{tr} \operatorname{res} M_{\mathscr{R}_{s}}^{(2)} \cdot \mathscr{R}_{r+n+1} d x=-\int \operatorname{tr} \operatorname{res} M_{\mathscr{R}_{s-n-1}}^{(1)} \cdot \mathscr{R}_{r+n+1} d x \\
& =\int \operatorname{tr} \operatorname{res} M_{\mathscr{R}_{r+n+1}}^{(1)} \cdot \mathscr{R}_{s-n-1} d x .
\end{aligned}
$$

Thus the subscript $r$ has increased by $n+1$ and the other subscript $s$ has decreased. One can continue this process until one of the subscripts becomes negative, and then $R_{k}=0$ for it.

Corollary. If one of the functionals $H_{r}$ is taken as a Hamiltonian then the other $H_{s}$ 's form an infinite set of first integrals in involution for the Hamiltonian equations (4.3)-(4.6).

Remark. Equation (4.5) for the Hamiltonian $H_{r}$ coincides with (4.6) for $H_{r+n+1}$.

\section{The Connection with Zakharov-Shabat Equations}

Let us consider (4.5) for the Hamiltonian $H_{r}$ in more detail. We have

$$
\dot{U}_{i}=\sum_{m=n-i}^{n}\left[U_{i+m-n}, R_{r+m-1}\right]+R_{r+n-i-1}^{\prime} ; \quad i=0, \ldots, n .
$$

Now $R_{k}$ are defined by the resolvent equation (5.2):

$$
R_{s-n-1}^{\prime}+\sum_{m=0}^{n+1}\left[U_{m}, R_{s+m-n-1}\right], \quad s=0,1,2, \ldots,\left(U_{n+1}=A\right) .
$$


If we take the first $r+n$ equations (9.2) then together with (9.1) they form a set of $r+2 n+1$ equations with the $r+2 n+1$ unknown functions $R_{0}, \ldots, R_{r+n-1}$, $U_{0}, \ldots, U_{n}$. This set is a particular case of the general Zakharov-Shabat scheme, see [2, p. 222]. Namely, if we consider an equation

$$
\dot{U}-V^{\prime}=[U, V],
$$

where

$$
\begin{aligned}
& U=U_{0}+\ldots+U_{n+1} \zeta^{n+1}, \\
& V=V_{0}+\ldots+V_{r+n-1} \zeta^{r+n-1},
\end{aligned}
$$

and if we require that the equation be satisfied identically with respect to $\zeta$, we shall obtain $r+2 n+1$ equations with $r+2 n+2$ unknown matrices. One of the matrices can be chosen arbitrarily. Let us take $U_{n+1}=A$. Then the set of the equations (9.1) and (9.2) $(s<r+n)$ is precisely obtained $\left(V_{k}=R_{r+n-1-k}\right)$.

There arises the question whether this set of equations is closed, i.e. whether all equations are independent. The answer is negative. Indeed, this set of equations has an additional degree of freedom, namely $n+1$ diagonal matrices can be chosen arbitrarily and only after this choice the set of the equations becomes definite. The situation can be explained in the following way. The $s^{\text {th }}$ equation (9.2) determines the nondiagonal part of $R_{s}$, and the diagonal part is only determined for $R_{s-n-1}$. For brevity, we omit the proof of this assertion. The same fact was encountered in Lemma 2 of Sect. 6 : to find the diagonal of $R_{0}$ we needed $n+2$ equations (6.7). The diagonal parts of the last $n+1$ matrices $R_{k}$ in the set (9.1) and (9.2) $(s<r+n)$ remain indeterminate. The best way to choose them is to use the next equations (9.2) without restricting ourselves by the condition $s<r+n$, i.e. to take as $R_{k}$ the coefficients of the resolvent, as was done in the previous sections.

We would like to emphasize that the question of definiteness of the ZakharovShabat equations must be treated very cautiously. They are definite as above, to within a single matrix, only if $U$ and $V$ have no common pole. Otherwise every common pole (with its multiplicity taken into account) brings an indeterminate diagonal matrix. The reason for this is just the same as for the fact that the equation $[B, U]=0$, where $B$ is a known and $U$ an unknown matrix is undetermined, though the number of equations is equal to the number of the unknown matrices, the degree of freedom reduces to the choice of a diagonal matrix. ${ }^{1}$

\section{Lagrangian}

Equation (4.6) will be derived from a variational principle. The general questions concerning the connections between the Hamiltonian and the Lagrangian approaches in similar problems will be discussed in a separate paper with Gelfand. It is yet unclear why this method fails in the case of Eq. (4.5).

We have the equation

$$
\dot{U}=M_{X}, \quad M_{X}=\tilde{\left[U+A \zeta^{n+1}, X\right]}, \quad\left(X=X_{f}\right) .
$$

1 Hamiltonian structures and integrable equations connected with scalar second order differential operators with polynomial dependence on a parameter were treated in [3] 
We reduce this equation to a matrix variety which can be written as

$$
U+A \zeta^{n+1}=(1+\varphi)^{-1} A \zeta^{n+1}(1+\varphi),
$$

where $\varphi=\sum_{0}^{n} \varphi_{i} \zeta^{-i-1}$. In the Kirillov-Kostant terminology this is an orbit of the coadjoint representation of the group $1+R_{-}$passing through the element $A \zeta^{n+1}$. It is easy to see that (10.2) imposes some requirements on $U$. For example, from $U_{n}=\left[A, \varphi_{0}\right]$ we see that the diagonal part of $U_{n}$ must vanish. However we shall see that the variety defined by (10.2) is compatible with (10.1).

Let $\mathscr{A}_{\varphi}$ be a differential algebra with independent differential generators which are elements of the matrices $\varphi_{i}$. According to (10.2) the algebra $\mathscr{A}$ is imbedded in $\mathscr{A}_{\varphi}$. Earlier the vector field $\xi_{M_{X}}=\xi_{X}$ acted in $\mathscr{A}$. Now we extend its action to the whole $\mathscr{A}_{\varphi}$. Its action on the generators $\varphi$ must be defined in such a way that (10.2) implies $\xi_{X} U=M_{X}$ [this will also prove the compatibility of the variety defined by (10.2) with (10.1)]. Let us apply $\xi_{X}$ to the right-hand side of (10.2):

$$
\begin{aligned}
& -(1+\varphi)^{-1} \xi_{X} \varphi(1+\varphi)^{-1} A \zeta^{n+1}(1+\varphi)+(1+\varphi)^{-1} A \zeta^{n+1} \xi_{X} \varphi \\
& =\left[U+A \zeta^{n+1},(1+\varphi)^{-1} \xi_{X} \varphi\right]=\left[U+\zeta^{n+1} A,(1+\varphi)^{-1} \xi_{X} \varphi\right] \text {. }
\end{aligned}
$$

It remains to put

$$
\overline{(1+\varphi)^{-1} \xi_{X} \varphi}=X
$$

and we receive $\xi_{X} U=\left[U+A \zeta^{n+1}, X\right]=M_{X}$. Now let

$$
\mathscr{L}=-f+\operatorname{tr} \operatorname{res}(1+\varphi)^{-1} A \zeta^{n+1} \dot{\varphi} .
$$

Theorem. Equation (10.1) is equivalent to the variational principle:

$$
\delta \mathscr{L} / \delta \varphi=0 .
$$

Proof. Taking into account (4.2) we have

$$
\begin{aligned}
\delta \mathscr{L}= & -\operatorname{tr} \operatorname{res} X_{f} \delta U+\operatorname{tr} \operatorname{res}\left(-(1+\varphi)^{-1} \delta \varphi(1+\varphi)^{-1} A \zeta^{n+1} \dot{\varphi}\right. \\
& \left.+(1+\varphi)^{-1} A \zeta^{n+1} \delta \dot{\varphi}\right)-\partial \omega_{s} \\
= & \operatorname{tr} \operatorname{res}\left\{X_{f}(1+\varphi)^{-1} \delta \varphi(1+\varphi)^{-1} A \zeta^{n+1}(1+\varphi)\right. \\
& -X_{f}(1+\varphi)^{-1} A \zeta^{n+1} \delta \varphi-(1+\varphi)^{-1} A \zeta^{n+1} \dot{\varphi}(1+\varphi)^{-1} \delta \varphi \\
& \left.+\partial_{t}\left((1+\varphi)^{-1} A \zeta^{n+1} \delta \varphi\right)+(1+\varphi)^{-1} \dot{\varphi}(1+\varphi)^{-1} A \zeta^{n+1} \delta \varphi\right\}-\partial \omega_{s} \\
= & \operatorname{tr} \operatorname{res}\left\{\left[U+A \zeta^{n+1}, X_{f}\right]-\dot{U}\right\}(1+\varphi)^{-1} \delta \varphi \\
& +\partial_{t} \omega_{n}-\partial \omega_{s} ; \quad \omega_{n}=\operatorname{tr} \operatorname{res}(1+\varphi)^{-1} A \zeta^{n+1} \delta \varphi, \quad \partial_{t}=\frac{\partial}{\partial t} .
\end{aligned}
$$

This implies

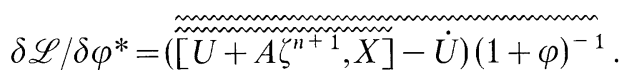

The rest is clear.

The formula (10.6) gives in fact more than a mere calculation of the variational derivative of $\mathscr{L}$. There are two more terms, namely, $-\partial \omega_{s}$ and $\partial \omega_{n}$ which play an important role. We have already mentioned that the form $\omega_{s}$ relates to stationary 
equations (see [1]). As to the second form, $\omega_{n}$, the integral $\omega=\int \delta \omega_{n} d x$ is the symplectic form, generated by the Lagrangian. We can prove directly by calculation that it coincides with the symplectic form $\omega_{2}$ (3.1): for any vector fields $\xi_{X}, \xi_{Y}$ we have

$$
\begin{aligned}
\omega\left(\xi_{X}, \xi_{Y}\right) & =-\int \operatorname{tr} \operatorname{res}(1+\varphi)^{-1} \delta \varphi(1+\varphi)^{-1} A \zeta^{n+1} \delta \varphi d x\left(\xi_{X}, \xi_{Y}\right) \\
& =-\int \operatorname{tr} \operatorname{res}(1+\varphi)^{-1} \delta \varphi\left(U+A \zeta^{n+1}\right)(1+\varphi)^{-1} \delta \varphi d x\left(\xi_{X}, \xi_{Y}\right) \\
& =-\int \operatorname{tr} \operatorname{res}(1+\varphi)^{-1} \xi_{X} \varphi\left(U+A \zeta^{n+1}\right)(1+\varphi)^{-1} \xi_{Y} \varphi d x-(X \leftrightarrow Y) \\
& =-\int \operatorname{tr} \operatorname{res} X\left(U+A \zeta^{n+1}\right) Y d x-(X \leftrightarrow Y) \\
& =\int \operatorname{tr} \operatorname{res}\left[U+A \zeta^{n+1}, X\right] Y d x=\omega_{2}\left(\xi_{X}, \xi_{Y}\right) .
\end{aligned}
$$

We could forget everything written earlier and start with the Lagrangian (10.4). We have derived Eq. (10.1) and the symplectic form from it, and we can also obtain the Hamiltonian or, even more, the energy-momentum tensor. The general rule for this is the following. Let $\mathscr{L}$ be an arbitrary Lagrangian which is a differential polynomial in $\varphi_{i}$. Then it can be proved that $\sum_{i} \varphi_{i, t} \delta \mathscr{L} / \delta \varphi_{i}$ and $\sum_{i} \varphi_{i, x} \delta \mathscr{L} / \delta \varphi_{i}$ are divergences of some vector fields whose components are also differential polynomials:

$$
-\sum \varphi_{i, t} \frac{\delta \mathscr{L}}{\delta \varphi_{i}}=\frac{\partial}{\partial t} T_{(t t)}+\frac{\partial}{\partial x} T_{(t x)}, \quad-\sum \varphi_{i, x} \frac{\delta \mathscr{L}}{\delta \varphi_{i}}=\frac{\partial}{\partial t} T_{(x t)}+\frac{\partial}{\partial x} T_{(x x)} .
$$

The quantities $T_{(t t)}, T_{(t x)}, T_{(x t)}, T_{(x x)}$ are the four components of the energymomentum tensor. Thus $H=\int T_{(t t)} d x$ is the Hamiltonian of the system. Let us find it for the Lagrangian (10.4)

$$
\begin{aligned}
\operatorname{mon}_{t}\left(T_{(t t)}\right)+\partial_{x}\left(T_{(t x)}\right) & =-\operatorname{tr} \operatorname{res} \dot{\varphi}\left(\left[U+A+A \zeta^{n+1}, X_{f}\right]-\dot{U}\right)(1+\varphi)^{-1} \\
& =-\operatorname{tr} \operatorname{res} \dot{\varphi}\left(\left[U+A \zeta^{n+1}, X_{f}\right]-\dot{U}\right)(1+\varphi)^{-1} .
\end{aligned}
$$

Then

$$
\begin{aligned}
& -\operatorname{tr} \operatorname{res} \dot{\varphi} \dot{U}(1+\varphi)^{-1}=-\operatorname{tr} \operatorname{res} \dot{\varphi}(1+\varphi)^{-1} A \zeta^{n+1} \dot{\varphi}(1+\varphi)^{-1} \\
& +\operatorname{tr} \operatorname{res} \dot{\varphi}(1+\varphi)^{-1} \dot{\varphi}(1+\varphi)^{-1} A \zeta^{n+1}(1+\varphi)(1+\varphi)^{-1}=0,
\end{aligned}
$$

and

$$
\begin{aligned}
-\operatorname{tr} & \operatorname{res} \dot{\varphi}\left[U+A \zeta^{n+1}, X_{f}\right](1+\varphi)^{-1} \\
= & -\operatorname{tr} \operatorname{res}\left\{(1+\varphi)^{-1} A \zeta^{n+1}(1+\varphi) X_{f}(1+\varphi)^{-1} \dot{\varphi}\right. \\
& \left.+X_{f}(1+\varphi)^{-1} A \zeta^{h+1}(1+\varphi)(1+\varphi)^{-1} \dot{\varphi}\right\}=\operatorname{tr} \operatorname{res} X_{f} \dot{U} \\
= & \sum_{i} \operatorname{tr} \frac{\delta f}{\delta U_{i}^{*}} \dot{U}_{i}=\sum_{i, k} \operatorname{tr} \frac{\partial f}{\partial U_{i}^{*(k)}} \dot{U}_{i}^{(k)}-\partial \sum_{i, k} \frac{\delta f}{\delta U_{i}^{*(k)}} \dot{U}^{(k-1)} \\
= & \partial_{t} f-\partial \sum_{i, k} \frac{\delta f}{\delta U_{i}^{*(k)}} \dot{U}^{(k-1)} .
\end{aligned}
$$

We have found two components of the energy-momentum tensor and $H=\int f d x$ as it should be expected. 


\section{Another Lagrangian}

Surprisingly, another, more sophisticated Lagrangian can be found. It leads to the same Hamiltonian structure (the symplectic form and the Hamiltonian). Its variational derivative is rather complicated, but equating it to zero we obtain an equation which is equivalent to the given one.

We start from the same equation (10.1) but instead of (10.2) we use another substitution. Now we shall write $V=\left(U+A \zeta^{n+1}\right) \zeta^{-n-1}=U \zeta^{-n-1}+A$. Then the equation is

$$
\dot{V}=\overline{[V, X]} \text {. }
$$

The substitution will be

$$
\zeta V_{\zeta}=\overline{[V, \varphi]}, \quad \varphi=\sum_{0}^{n} \varphi_{i} \zeta^{-i-1},
$$

(the subscript $\zeta$ denotes the derivative). In more detail the equation is written as

$$
\begin{aligned}
\dot{U}_{0}= & {\left[U_{1}, X_{0}\right]+\left[U_{2}, X_{1}\right]+\ldots+\left[A, X_{n}\right], } \\
\dot{U}_{1}= & {\left[U_{2}, X_{0}\right]+\ldots+\left[A, X_{n-1}\right], } \\
& \ldots \\
\dot{U}_{n}= & {\left[A, X_{0}\right], }
\end{aligned}
$$

and the substitution is

$$
\begin{aligned}
U_{0}= & -\frac{1}{n+1}\left(\left[U_{1}, \varphi_{0}\right]+\ldots+\left[A, \varphi_{n}\right]\right), \\
U_{1}= & -\frac{1}{n}\left(\left[U_{2}, \varphi_{0}\right]+\ldots+\left[A, \varphi_{n-1}\right]\right), \\
& \ldots \\
U_{n}= & -\left[A, \varphi_{0}\right] .
\end{aligned}
$$

It is clear that not every set of matrices $U_{i}$ can be presented in the form (11.2). For example, as follows from the last of the equations (11.4), the diagonal of $U_{n}$ must be zero. It can be proved that (11.2) or (11.4) is equivalent to the following condition on $U_{i}$ : the diagonal of $U_{i}$ is expressible in terms of nondiagonal parts of $U_{k}$ with $k>i$. Thus (11.2) or (11.4) specifies a variety of matrices. We shall see that this variety is invariant under the vector field (11.1).

The substitution (11.2) gives an imbedding of the differential algebra $\mathscr{A}$ in an algebra $\mathscr{A}_{\varphi}$ which is generated by the elements of $\varphi_{i}$. Now we define a vector field $\xi$ in $\mathscr{A}_{\varphi}$ in such a way that its action on the variety defined by (11.2) coincides with $\xi_{X}: \xi_{X} V=\overline{[V, X]}$. This will mean that $\xi_{X}$ is tangent to this variety and thus the variety is compatible with the equation. Let us put

$$
\xi \varphi=\zeta X_{\zeta}-\overline{[X, \varphi]} .
$$

Proposition. $\xi V=\overline{[V, X]}$. 
Proof. We apply the differentiation $\xi$ to (11.2):

$$
\begin{aligned}
\zeta(\xi V)_{\zeta} & =\overline{[\xi V, \varphi]}+\overline{[V, \xi \varphi]}=\overline{[\xi V, \varphi]}+\overline{\left[V, \zeta X_{\zeta}\right]}-\overline{[V, \overline{[X, \varphi]}} \\
& =\overline{[\xi V, \varphi]}+\overline{\left[V, \zeta X_{\zeta}\right.}+\overline{[\varphi,[V, X]]}+\overline{[X,[\varphi, V]]} \\
& =\overline{[\xi V-\overline{[V, X]}, \varphi]}+\overline{\left[V, \zeta X_{\zeta}\right]}-\overline{\left[X, \zeta V_{\zeta}\right]} \\
& =\overline{[\xi V-\overline{[V, X]}, \varphi]}+\overline{\zeta[V, X]_{\zeta}}
\end{aligned}
$$

or, denoting $\xi V-\overline{[V, X]}=W$,

$$
\zeta W_{\zeta}-\overline{[W, \varphi]}=0 \text {. }
$$

The last equation has the same form as (11.2). However there is a distinction between $W$ and $V$ :

$$
V=A+U_{0} \zeta^{-1}+\ldots+U_{n} \zeta^{-n-1} \text { and } W=W_{0} \zeta^{-1}+\ldots+W_{n} \zeta^{-n-1} .
$$

Therefore $W_{i}$ satisfy (11.4) with $A=0$. This immediately gives $W=0$ and $\xi V=\overline{[V, X]}$ as required.

Now we put

$$
\mathscr{L}=-f-\operatorname{tr} \frac{1}{n+2} \sum_{i=0}^{n} U_{i} \dot{\varphi}_{i}
$$

This expression will be taken as a Lagrangian. Let $O_{j}=\sum_{k=0}^{n-j}\left[U_{k+1+j}, X_{k}\right]-\dot{U}_{j}[$ the differences between the left-hand and the right-hand sides of (11.3)].

\section{Theorem.}

$$
\begin{aligned}
\delta \mathscr{L}= & \left.\left.-\operatorname{tr} \sum_{i=0}^{n} \sum_{(\alpha)} c_{i(\alpha)}\left[\ldots\left[Q_{i+r+\alpha_{1}+\ldots+\alpha_{r}}, \varphi_{\alpha_{1}}\right], \varphi_{\alpha_{2}}\right], \ldots\right], \varphi_{\alpha_{r}}\right] \delta \varphi_{i} \\
& -\partial \omega_{s}-\partial_{t} \frac{1}{n+2} \operatorname{tr} \sum_{i} U_{i} \delta \varphi_{i},
\end{aligned}
$$

where the summation extends over all the sets of nonnegative integers $(\alpha)=\left(\alpha_{1}, \ldots, \alpha_{r}\right)$ for which $i+r+\alpha_{1}+\ldots+\alpha_{r} \leqq n$ (the order of the integers is important here), $\omega_{s}$ is the same 1-form as in Sect. 10 and the coefficients $c_{i(\alpha)}$ are

$$
\begin{aligned}
c_{i(\alpha)}= & {\left[\left(1+i+r+\alpha_{1}+\ldots+\alpha_{r}\right)\left(i+r+\alpha_{2}+\ldots+\alpha_{r}\right)\left(i-1+r+\alpha_{3}+\ldots+\alpha_{2}\right)\right.} \\
& \left.\ldots\left(i+2+\alpha_{r}\right)(i+1)\right]^{-1} .
\end{aligned}
$$

If $r=0$ then $c_{i(\alpha)}=(i+1)^{-1}$.

Corollary. The Euler-Lagrange set of equations

$$
\delta \mathscr{L} / \delta \varphi_{k}=0, \quad k=0,1, \ldots, n
$$

is equivalent to (11.3).

Only a brief sketch of the proof will be given. Recall that $X=X_{f}$ and $X_{k}=\delta f / \delta U_{k}^{*}$. We have

$$
\delta f=\operatorname{tr} \sum \delta f / \delta U_{k}^{*} \cdot \delta U_{k}+\delta \omega_{s}
$$


It is necessary to transform $\operatorname{tr} \sum X_{k} \delta U_{k}$ to the form $\operatorname{tr} \sum a_{k} \delta \varphi_{k}$. Let

$$
\operatorname{tr} g \delta U_{k}=\operatorname{tr} \sum_{i=0}^{n-k} a_{k i}(g) \delta \varphi_{i}
$$

Lemma 1. The coefficients $a_{k i}$ satisfy the set of recurrence equations

$$
a_{k i}(g)=(n+1-k)^{-1}\left\{\sum_{j=0}^{n-k-i-1} a_{k+j+1, i}\left(\left[g, \varphi_{j}\right]\right)+\left[U_{k+i+1}, g\right]\right\} .
$$

Here we put $U_{n+1}=A$.

To prove this lemma it is necessary to substitute $\delta U_{k}$ from (11.4) into the lefthand side of (11.6), to express all the summands with $\delta U_{i}$ in terms of $\delta \varphi_{i}$ using (11.6) and to equate the summands with the same $\delta \varphi_{i}$.

Remark. Equation (11.7) enables us to determine all the coefficients $a_{k i}$ : first $a_{n-i, i}=(1+i)^{-1}[A, g]$, then $a_{n-i-1, i}$ etc.

\section{Lemma 2.}

$$
\left.\left.a_{k i}(g)=-\sum_{(\alpha)} c_{i(\alpha)}\left[\ldots\left[g, U_{k+1+i+r+\alpha_{1}+\ldots+\alpha_{r}}\right], \varphi_{\alpha_{1}}\right], \ldots, \varphi_{\alpha_{r-1}}\right], \varphi_{\alpha_{r}}\right]
$$

$\left(k+1+i+r+\alpha_{1}+\ldots+\alpha_{r} \leqq n+1\right.$ and the coefficients $c_{i(\alpha)}$ are given above).

To prove this lemma one has to check that the expression (11.8) satisfies (11.7).

Now let us prove the theorem. We have

$$
\begin{aligned}
-\delta \mathscr{L}= & \operatorname{tr} \sum_{0}^{n} X_{k} \delta U_{k}+\partial \omega_{s}-\frac{1}{n+2} \operatorname{tr} \sum \dot{U}_{k} \delta \varphi_{k}+\frac{1}{n+2} \operatorname{tr} \sum \dot{\varphi}_{k} \delta U_{k} \\
& +\partial_{t} \frac{1}{n+2} \operatorname{tr} \sum U_{k} \delta \varphi_{k} \\
= & \operatorname{tr} \sum_{k=0}^{n} \sum_{i=0}^{n} a_{k i}\left(X_{k}+\frac{1}{n+2} \dot{\varphi}_{k}\right) \delta \varphi_{i}-\frac{1}{n+2} \operatorname{tr} \sum \dot{U}_{k} \delta \varphi_{k} \\
& +\partial \omega_{s}+\partial_{t} \operatorname{tr} \frac{1}{n+2} \sum U_{k} \delta \varphi_{k} .
\end{aligned}
$$

Further,

$$
\begin{aligned}
\sum_{k=0}^{n} a_{k i}\left(\dot{\varphi}_{k}\right)= & \left.-\sum_{(\alpha), k} c_{i(\alpha)}\left[\ldots\left[\dot{\varphi}_{k}, U_{k+1+i+r+\alpha_{1}+\ldots+\alpha_{r}}\right], \varphi_{\alpha_{1}}\right], \ldots\right] \\
= & \left.-\sum_{(\alpha), k} c_{i(\alpha)}\left[\ldots\left[\varphi_{k}, U_{k+1+i+r+\alpha_{1}+\ldots+\alpha_{r}}\right], \varphi_{\alpha_{1}}\right] \ldots\right] \\
& \left.+\sum_{(\alpha), k} c_{i(\alpha)}\left[\ldots\left[\varphi_{k}, \dot{U}_{k+1+i+r+\alpha_{1}+. .+\alpha_{r}}\right], \varphi_{\alpha_{1}}\right] \ldots\right]
\end{aligned}
$$

or, taking into account (11.4),

$$
\begin{aligned}
& -\sum_{(\alpha)} c_{i(\alpha)}\left(n+1-i-r-\alpha_{1}-\ldots-\alpha_{r}\right)\left[\ldots\left[\dot{U}_{i+r+\alpha_{1}+\ldots+\alpha_{r}}, \varphi_{\alpha_{1}}\right] \ldots\right] \\
& \left.-\sum_{(\alpha), k} c_{i(\alpha)}\left[\ldots\left[\dot{U}_{k+1+i+r+\alpha_{1}+\ldots+\alpha_{r}}, \varphi_{k}\right], \varphi_{\alpha_{1}}\right], \ldots\right] \\
= & -(n+2) \sum_{(\alpha)} c_{i(\alpha)}\left[\ldots\left[\dot{U}_{i+r+\alpha_{1}}+\ldots+\alpha_{r}, \varphi_{\alpha_{1}}\right] \ldots\right]+\dot{U}_{i} .
\end{aligned}
$$


Now

$$
\begin{aligned}
\delta \mathscr{L}= & \operatorname{tr} \sum_{i=0}^{n} \sum_{(\alpha)} c_{i(\alpha)}\left\{\sum_{k}\left[\ldots\left[X_{k}, U_{k+1+i+r+\alpha_{1}+\ldots+\alpha_{r}}\right], \varphi_{\alpha_{1}}\right] \ldots\right] \\
& \left.+\left[\ldots\left[\dot{U}_{i+r+\alpha_{1}+\ldots+\alpha_{r}}, \varphi_{\alpha_{1}}\right] \ldots\right]\right\} \delta \varphi_{i}-\partial \omega_{s}-\partial_{t} \frac{1}{n+2} \sum U_{k} \delta \varphi_{k} \\
= & -\operatorname{tr} \sum_{i=0}^{n} \sum_{(\alpha)} c_{i(\alpha)}\left[\ldots\left[Q_{i+r+\alpha_{1}+\ldots+\alpha_{r}}, \varphi_{\alpha_{1}}\right], \ldots\right] \delta \varphi_{i} \\
& -\partial \omega_{s}-\partial_{t} \frac{1}{n+2} \operatorname{tr} \sum U_{k} \delta \varphi_{k}
\end{aligned}
$$

as stated.

The expressions for the variational derivatives of $\mathscr{L}$ are

$$
\frac{\delta \mathscr{L}}{\delta \varphi_{i}^{*}}=-\sum_{(\alpha)} c_{i(\alpha)}\left[\ldots\left[Q_{i+r+\alpha_{1}+\ldots+\alpha_{r}}, \varphi_{\alpha_{1}}\right] \ldots\right] .
$$

This proves the corollary: if $\delta \mathscr{L} / \delta \varphi_{i}^{*}=0$ we get in succession $Q_{n-1}=0, Q_{n-2}=0$, etc.

Proposition. The 1-form

$$
\omega=-\frac{1}{n+2} \int \operatorname{tr} \sum \delta U_{k} \delta \varphi_{k} d x=-\frac{1}{n+2} \int \operatorname{tr} \operatorname{res} \delta U \delta \varphi d x
$$

coincides with $\omega_{2}$ in (3.1) on the vector fields tangent to the variety defined by (11.2).

Proof. We have

$$
\begin{aligned}
(n+ & 2) \omega\left(\xi_{X}, \xi_{Y}\right)=\int \operatorname{tr} \operatorname{res}\left(-\xi_{X} U \cdot \xi_{Y} \varphi+\xi_{Y} U \cdot \xi_{X} \varphi\right) d x \\
& =\int \operatorname{tr} \operatorname{res} \zeta^{n+1}\left(-\xi_{X} V \cdot \xi_{Y} \varphi+\xi_{Y} V \cdot \xi_{X} \varphi\right) d x \\
& =\int \operatorname{tr} \operatorname{res} \zeta^{n+1}\left\{\overline{[V, X]}\left(-\zeta Y_{\zeta}+\overline{[Y, \varphi]}\right)-\overline{[V, Y]}\left(-\zeta X_{\zeta}+\overline{[X, \varphi]}\right)\right\} d x \\
& =\int \operatorname{tr} \operatorname{res} \zeta^{n+1}\left\{[V, X]\left(-\zeta Y_{\zeta}+[Y, \varphi]\right)-[V, Y]\left(-\zeta X_{\zeta}+[X, \varphi]\right)\right\} d x .
\end{aligned}
$$

Now

$$
\operatorname{tr}([V, X][Y, \varphi]-[V, Y][X, \varphi])=\operatorname{tr} Y[X,[V, \varphi]] .
$$

Therefore, from (11.2):

$$
\begin{aligned}
(n+2) \omega\left(\xi_{X}, \xi_{Y}\right) & =\int \operatorname{tr} \operatorname{res} \zeta^{n+1}\left\{-[V, X] \zeta Y_{\zeta}+[V, Y] \zeta X_{\zeta}+Y\left[X, \zeta V_{\zeta}\right]\right\} \\
& =-\int \operatorname{tr} \operatorname{res} \zeta^{n+1} \zeta \frac{\partial}{\partial \zeta}[V, X] Y d x \\
& =(n+2) \int \operatorname{tr} \operatorname{res} \zeta^{n+1}[V, X] Y d x \\
& =(n+2) \int \operatorname{tr} \operatorname{res}\left[U+A \zeta^{n+1}, X\right] Y d x \\
& =(n+2) \int \operatorname{tr} \operatorname{res}\left[U+A \zeta^{n+1}, X\right] Y d x \\
& =(n+2) \omega_{2}\left(\xi_{X}, \xi_{Y}\right) .
\end{aligned}
$$


It is unnecessary to verify that the Hamiltonian will be the same as before, i.e. $H=\int f d x$. This follows automatically from the coincidence of the equation and the symplectic form.

\section{References}

1. Dickey, L.A.: Integrable nonlinear equations and Liouville's theorem, I and II. Commun. Math. Phys. 82, 345-360 and 361-375 (1981)

2. Novikov, S.P. (ed.): Soliton theory. Moscow: Nauka 1980 (in Russian)

3. Martinez Alonso, L.: Gel'fand-Dikii method and nonlinear equations associated to Schrödinger operators with energy-dependent potentials. Lett. Math. Phys. 4, 177-184 (1980)

Communicated by A. Jaffe

Received January 12, 1982 